\title{
A RELATION BETWEEN THE CYLINDRIC FLUID MEMBRANES AND THE MOTIONS OF PLANAR CURVES
}

\author{
PETKO I. MARINOV AND IVAÏLO M. MLADENOV
}

Presented by Ivaïlo M. Mladenov

Abstract. We observe a relation between the mKdV equation and the cylindrical equilibrium shapes of fluid membranes. In our setup $\mathrm{mKdV}$ arises from the study of the evolution of planar curves.

\section{Introduction}

The goal of this paper is to unify and extend the results presented in [5] and [8]. It also shows a connection between two problems that appear unrelated.

The first problem comes from the study of equilibrium shapes of fluid membranes. One starts with a functional proposed by Helfrich (see [2], [8]) and studies the corresponding Euler-Lagrange equation. The equilibrium shapes are given as the extremals of the functional

$$
\mathcal{F}=\frac{k_{c}}{2} \int_{S}(2 H+\mathrm{h})^{2} \mathrm{~d} A+k_{G} \int_{S} K \mathrm{~d} A+\lambda \int_{S} \mathrm{~d} A+p \int \mathrm{d} V .
$$

Notice that $\mathcal{F}$ is closely related to the Willmore energy functional. The EulerLagrange equation associated with $\mathcal{F}$ is as follows

$$
2 k_{c} \Delta_{S} H+k_{c}(2 H+\mathrm{h})\left(2 H^{2}-\mathrm{h} H-2 K\right)-2 \lambda H+p=0 .
$$

Here $H$ and $K$ are the mean and Gauss curvatures respectively, $k_{c}$ and $k_{G}$ - bending and Gaussian constant rigidity of the membrane, $\mathrm{th}$ is spontaneous curvature constant, $p$ and $\lambda$ - Lagrange multipliers corresponding to fixed volume and total membrane area and $\Delta_{S}$ is the surface Laplacian on the interface of the membrane. The nature of this equation is complex as it involves the surface Laplacian of the mean curvature which makes it a fourth-order non-linear PDE. However, as always, the symmetry of the problem reduces the equation and in the special case of cylindrical membranes it becomes the ordinary differential equation

$$
2 \frac{\mathrm{d}^{2} \kappa}{\mathrm{d} s^{2}}+\kappa^{3}-\mu \kappa-\sigma=0 .
$$


Here $\kappa=\kappa(s)$ is a curvature of a planar curve and $\sigma, \mu$ are physical parameters, more precisely

$$
\mu=\mathbb{h}^{2}+\frac{2 \lambda}{k_{c}}, \quad \sigma=-\frac{2 p}{k_{c}} .
$$

This curve is the directrix of the cylindrical fluid membrane we look at. The equation (3) can be integrated once and we get

$$
\left(\frac{\mathrm{d} \kappa}{\mathrm{d} s}\right)^{2}=P(\kappa)
$$

where $P(\kappa)$ is a fourth degree polynomial in $\kappa$ with zero cubic term. The above equation is solved for all cases of interest depending on the roots of $P(\kappa)$ in [8]. Obviously, the roots add up to zero.

The key observation in the present paper is that (4) appears in the study of the motion of curves in the plane and the modified $\mathrm{KdV}(\mathrm{mKdV})$ equation. In [6] the soliton approach gives the dynamics of the curvature of an elastic rod, which yields the mKdV hierarchy while [5] focuses on general motion of a planar curve, which gives raise to the same considerations presented in [6]. Following [6] we consider the general evolution of a curve in the plane, which is given by

$$
\frac{\mathrm{d} \mathbf{x}}{\mathrm{d} s}=U \mathbf{t}+W \mathbf{n}
$$

where $\mathbf{x}=\mathbf{x}(s, t)$ is the position vector in the plane, $\mathbf{n} a n d \mathbf{t}$ are the unit normal and the unit tangent to the curve at given time $t$ and $U, W$ are certain velocities that are determined by the curvature of the curve. The evolution of the curvature itself is given and derived by the equation

$$
\frac{\partial \kappa}{\partial t}=\frac{\partial^{2} W}{\partial s^{2}}+\kappa^{2} W+\frac{\partial \kappa}{\partial s} \int k W \mathrm{~d} s \equiv R W .
$$

These considerations for the evolution of the curvature yield the $\mathrm{mKdV}$ equation (see [5], [6], [7]) yeld

$$
\frac{\partial \kappa}{\partial t}-\frac{\partial^{3} \kappa}{\partial s^{3}}-\frac{3}{2} \kappa^{2} \frac{\partial \kappa}{\partial s}=0
$$

We get the mKdV by setting $W$ to be $\frac{\partial \kappa}{\partial s}$ assuming that $\kappa(s, t)$ is the curvature of the members of a family of evolving planar curves.

Setting $\kappa=\kappa(s-\lambda t)$, we get immediately an ODE, which after two integrations becomes (4). Therefore the ODE version of (6) is the same as the symmetric version of the Euler-Lagrange equation for the elastic membranes. Some preliminary calculations for the most simple cases of the roots of $P(\kappa)$ are presented in [5]. 
Here we will carry out the calculations in all relevant cases. From [8] or [7] we get formulas to represent the solutions and in the main section of this paper we will derive the analytical solutions of (4) in order to get all planar motions of the family of evolving curves. The relation between the Gardner equation and the traveling wave solutions of the mKdV curve flow is studied in [7]. This is an evidence that a relation of this nature appears frequently.

In addition to the computations done here we also show the phase portrait for all relevant cases for the roots of $P(\kappa)$ which presents an interest of its own. For example phase portraits are also shown in [1]. Both the computations and the pictures presented in this paper are done by using Maple 8 software. Some flow animation for the solutions of the $\mathrm{mKdV}$ equation was also done but naturally it cannot be presented here.

Another important observation regarding this paper is that it also hints a relation between the objects described above and the so called Euler elastica, where the energy of the configuration in question is again given as the integral of the curvature squared (see [10]).

\section{Main Results}

In this section we will present our main results. There are exactly three relevant cases deserving consideration, depending on the roots of the polynomial $P(\kappa)$. We work in the case $\sigma \neq 0$ (it can be done in the zero case too) and we use a formula from [8], namely

$$
\begin{aligned}
& x(s)=\frac{2}{\sigma} \frac{\mathrm{d} \kappa(s)}{\mathrm{d} s} \cos \theta(s)+\frac{1}{\sigma}\left(\kappa^{2}(s)-\mu\right) \sin \theta(s) \\
& z(s)=\frac{2}{\sigma} \frac{\mathrm{d} \kappa(s)}{\mathrm{d} s} \sin \theta(s)-\frac{1}{\sigma}\left(\kappa^{2}(s)-\mu\right) \cos \theta(s) .
\end{aligned}
$$

Therefore we need the angle of inclination and curvature (also given in [8]).

Case 1. In the following calculations we assume that $P(\kappa)$ has two real roots $\alpha<\beta$ and a pair of complex roots $\gamma, \bar{\gamma}$ which obey to $(3 \alpha+\beta)(\alpha+3 \beta) \neq 0$. The roots must add up to zero. This is the case treated in [8], namely in the first part of Theorem 1. Assuming $\sigma \neq 0$ (one can do it for $\sigma=0$ too but we need to use different set of formulae from [8]) we get the angle on inclination and the curvature in terms of the Jacobian elliptic functions as follows

$$
\kappa_{1}(s)=\frac{A \beta+B \alpha-(A \beta-B \alpha) \operatorname{cn}(u s, k)}{A+B-(A-B) \operatorname{cn}(u s, k)}
$$




$$
\begin{aligned}
\theta_{1}(s)= & \frac{(A \beta-B \alpha) s}{A-B}+\frac{(A+B)(-\beta+\alpha)}{2 u(A-B)} \Pi\left(\operatorname{sn}(u s, k),-\frac{(A-B)^{2}}{4 B A}, k\right) \\
& +\frac{\alpha-\beta}{u \sqrt{4 k^{2}+\frac{(A-B)^{2}}{B A}}} \arctan \left(\sqrt{k^{2}+\frac{(A-B)^{2}}{4 B A}} \frac{\operatorname{sn}(u s, k)}{\operatorname{dn}(u s, k)}\right) .
\end{aligned}
$$

We use standard notation: $\operatorname{sn}(x, k), \operatorname{cn}(x, k), \operatorname{dn}(x, k)$ and $\Pi(\operatorname{sn}(x, k), n, k)$ are the Jacobian elliptic functions and the elliptic integral of the third kind with elliptic modulus $k$ (one may look for details at [3]). It is convenient to introduce

$$
A=\sqrt{4 \eta^{2}+(3 \alpha+\beta)^{2}}, \quad B=\sqrt{4 \eta^{2}+(\alpha+3 \beta)^{2}}, \quad u=1 / 4 \sqrt{A B}
$$

with $\eta$ being the imaginary part of $\gamma$. Also, $k$ is defined as

$$
k=\frac{1}{\sqrt{2}} \sqrt{1-\frac{4 \eta^{2}+(3 \alpha+\beta)(\alpha+3 \beta)}{\left(4 \eta^{2}+(3 \alpha+\beta)(\alpha+3 \beta)\right)^{2}+16 \eta^{2}(\beta-\alpha)^{2}}} .
$$

Let us set

$$
\begin{gathered}
\lambda=\sqrt{4 k^{2}+\frac{(A-B)^{2}}{B A}} \\
\operatorname{sn}(s)=\operatorname{sn}(u s, k), \quad \operatorname{cn}(s)=\operatorname{cn}(u s, k), \quad \operatorname{dn}(s)=\operatorname{dn}(u s, k)
\end{gathered}
$$

and

$$
\tilde{\Pi}(s)=\Pi\left(\operatorname{s} \tilde{n}(s),-\frac{(A-B)^{2}}{4 B A}, k\right) .
$$

Using this notation we compute the profile curve from (7). Let us set the derivative and the square of the curvature to be respectively

$$
\begin{aligned}
A_{1}= & \frac{(A \beta-B \alpha) u \mathrm{~d} \tilde{\mathrm{n}}(s) \mathrm{s} \tilde{\mathrm{n}}(s)}{A+B-(A-B) \mathrm{c} \tilde{\mathrm{n}}(s)} \\
& -\frac{(A \beta+B \alpha-(A \beta-B \alpha) \mathrm{c} \tilde{\mathrm{n}}(s)) u(A-B) \mathrm{d} \tilde{\mathrm{n}}(s) \mathrm{s} \tilde{n}(s)}{(A+B-(A-B) \mathrm{c} \tilde{\mathrm{n}}(s))^{2}} \\
B_{1}= & \frac{(A \beta+B \alpha-(A \beta-B \alpha) \mathrm{c} \tilde{\mathrm{n}}(s))^{2}}{(A+B-(A-B) \mathrm{c} \tilde{\mathrm{n}}(s))^{2}} .
\end{aligned}
$$

Then we get the representation of the solution curve

$$
\begin{aligned}
& x_{1}(s)=\frac{2}{\sigma} A_{1} \cos \left(\theta_{1}\right)+\frac{1}{\sigma}\left(B_{1}-\mu\right) \sin \left(\theta_{1}\right) \\
& z_{1}(s)=\frac{2}{\sigma} A_{1} \sin \left(\theta_{1}\right)-\frac{1}{\sigma}\left(B_{1}-\mu\right) \cos \left(\theta_{1}\right) .
\end{aligned}
$$



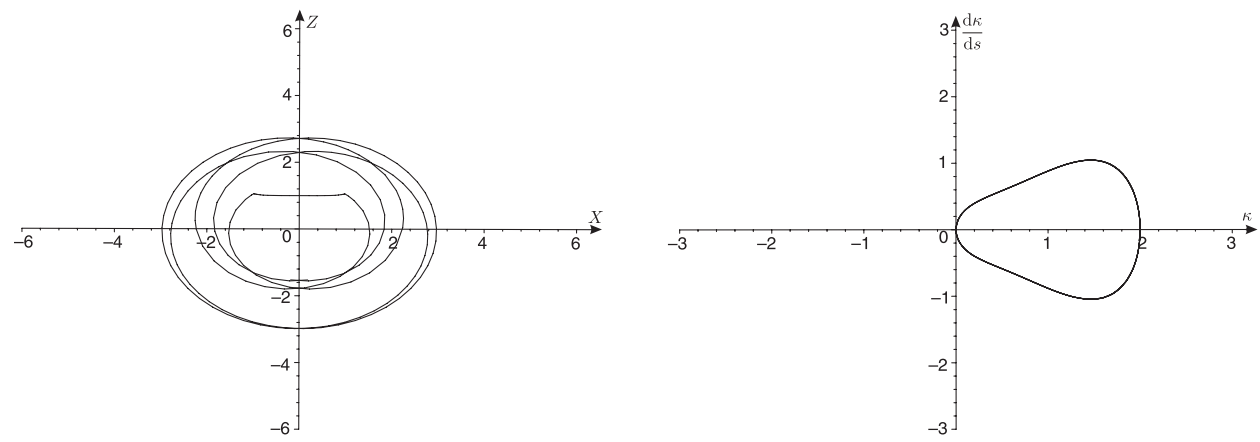

Figure 1. Solution curve (left) and phase portrait (right) for $\alpha=0, \beta=2$, $\gamma=-1-\mathrm{i}$.

Here is an example. Pick $A=\sqrt{8}, B=\sqrt{40}, \alpha=0, \beta=2, u=\sqrt{\sqrt{320}}$ and $m=\sqrt{190} / 20$. These values $(A, B$, etc. ) are obtained by picking $\gamma=-1-\mathrm{i}$ and $\bar{\gamma}=-1+\mathrm{i}$. Notice that the condition $\alpha+\beta+\gamma+\bar{\gamma}=0$ is fulfilled, which was necessary. The solution curve and the phase portrait corresponding to the above example are depicted in Fig. 1. On the left is given the curve $\left(x_{1}(s), z_{1}(s)\right)$ and on the right is $\left(\kappa_{1}(s), \frac{\mathrm{d} \kappa_{1}(s)}{\mathrm{d} s}\right)$.

Case 2. Next is the easiest case described in ([8], second part of Theorem 1). Here the polynomial $P(\kappa)$ has two real roots $\alpha<\beta$ and a pair of complex roots $\gamma, \bar{\gamma}$ with $(3 \alpha+\beta)(\alpha+3 \beta)=0$. Let $\xi=\alpha$ if $3 \alpha+\beta=0$ and $\xi=\beta$ otherwise. Again we need the roots to sum up to zero. These two conditions actually imply that $\sigma \neq 0$. Now one has the following expressions for the curvature and the angle

$$
\kappa_{2}(s)=\xi-4 \frac{\xi}{1+\xi^{2} s^{2}}, \quad \theta_{2}(s)=\xi s-4 \arctan (\xi s) .
$$

Because $\sigma \neq 0$ and following [8] we get the following representation for the directrix using (7)

$$
\begin{aligned}
& x_{2}(s)=\frac{2}{\sigma} A_{2} \cos \left(\theta_{2}\right)+\frac{1}{\sigma}\left(B_{2}-\mu\right) \sin \left(\theta_{2}\right) \\
& z_{2}(s)=\frac{2}{\sigma} A_{2} \sin \left(\theta_{2}\right)-\frac{2}{\sigma}\left(B_{2}-\mu\right) \cos \left(\theta_{2}\right)
\end{aligned}
$$

where

$$
\begin{aligned}
& A_{2}=16 \frac{\xi^{3} s \cos (\xi s-4 \arctan (\xi s))}{\sigma\left(1+\xi^{2} s^{2}\right)^{2}} \\
& B_{2}=\left(\xi-4 \frac{\xi}{1+\xi^{2} s^{2}}\right)^{2} .
\end{aligned}
$$



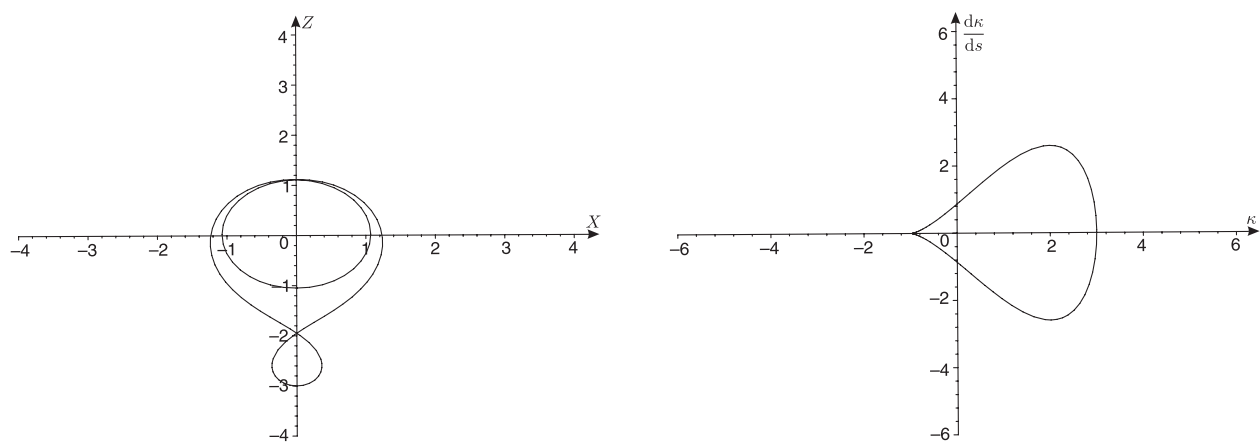

Figure 2. Solution curve (left) and the phase portrait (right) for $\alpha=\gamma=\bar{\gamma}$ $=-1$ and $\beta=3$.

For example if the roots are $\{-1,-1,-1,3\}$ we have $\xi=-1, \mu=3$ and $\sigma=2$ and we get the directrix depicted in Fig. 2. We get the flow corresponding to this case if we substitute $s-c t$ for $s$ in (14). One can also get the phase portrait of this case (see Fig. 2, right part).

Case 3. In the last case we will consider the polynomial $P(\kappa)$ with four real roots $\alpha<\beta<\gamma<\delta$. One possible solution (i.e., the curvature, angle and coordinate functions for the directrix) is given below. The other one is described in [8] and has very similar structure. For that one we will only give the angle and curvature as is given in [8]. Again a phase portrait is given for a particular choice of values for the roots of $P(\kappa)$.

Let

$$
\begin{gathered}
p=\frac{(\gamma-\alpha)(\delta-\beta)}{4}, \quad q=\sqrt{\frac{(\beta-\alpha)(\delta-\gamma)}{(\gamma-\alpha)(\delta-\beta)}} \\
\operatorname{cn}(s)=\operatorname{cn}(p s, q), \quad \operatorname{dn}(s)=\operatorname{dn}(p s, q), \quad \sin (s)=\operatorname{sn}(p s, q) .
\end{gathered}
$$

Using this notation we get the two curvatures and the two angles corresponding to this case

$$
\begin{aligned}
& \kappa_{3}(s)=\delta-(\delta-\alpha)(\delta-\beta)\left(\delta-\beta+(\beta-\alpha) \hat{s}^{2}(s)\right)^{-1} \\
\theta_{3}(s)= & \delta s-4 \Pi\left(\sin (s), \frac{\beta-\alpha}{\beta-\delta}, q\right)(\delta-\alpha)(\gamma-\alpha)^{-1 / 2}(\delta-\beta)^{-1 / 2} \\
& \kappa_{4}(s)=\beta+(\gamma-\beta)(\delta-\beta)\left(\delta-\beta-(\delta-\gamma) \operatorname{s\hat {n}}^{2}(s)\right)^{-1} \\
\theta_{4}(s)= & \beta s-4 \Pi\left(\sin (s), \frac{\delta-\gamma}{\delta-\beta}, q\right)(\beta-\gamma)(\gamma-\alpha)^{-1 / 2}(\delta-\beta)^{-1 / 2} .
\end{aligned}
$$



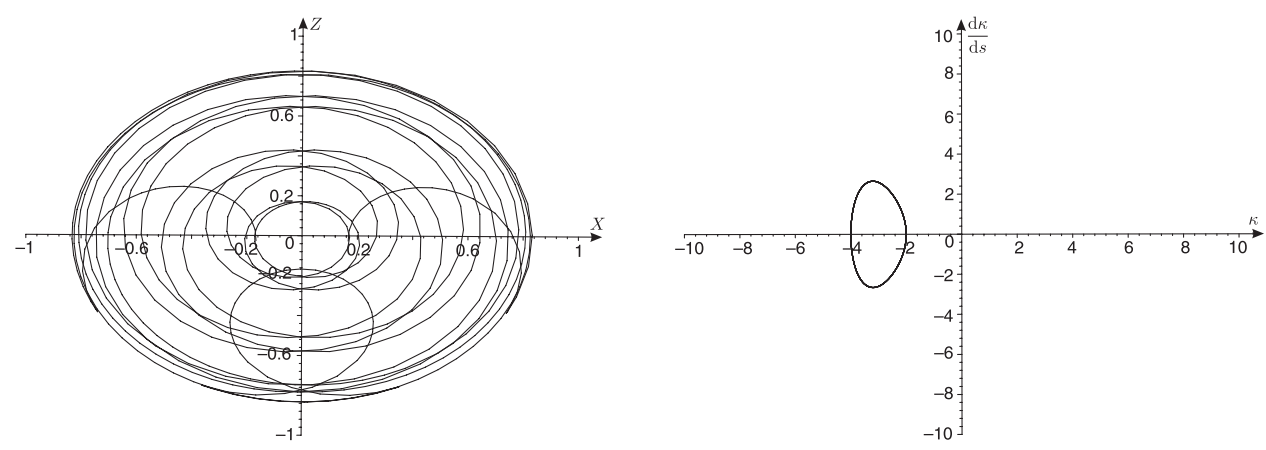

Figure 3. Solution curve (left) and phase portrait (right) for $\alpha=-4, \beta=-2$, $\gamma=0$ and $\delta=6$.

Now, we apply again formula (7) for the solution curve to obtain $x_{3}(s)$ and $z_{3}(s)$. Similarly one can get formula corresponding to $\kappa_{4}(s)$ and $\theta_{4}(s)$. Again we assume that $\sigma \neq 0$. As in the previous cases we will set up two quantities $A_{3}$ and $B_{3}$.

$$
\begin{aligned}
& A_{3}=\frac{(\delta-\alpha)(\delta-\beta)^{3 / 2}(\gamma-\alpha)^{1 / 2}(\beta-\alpha)}{\left(\delta-\beta+(\beta-\alpha) \sin ^{2}(s)\right)^{2}} \sin (s) \operatorname{con}(s) \mathrm{d} \hat{n}(s) \\
& B_{3}=\left(\delta-(\delta-\alpha)(\delta-\beta)\left(\delta-\beta+(\beta-\alpha) \sin ^{2}(s)\right)^{-1}\right)^{2} .
\end{aligned}
$$

Lastly, we get the formulae for the profile curve in this case

$$
\begin{aligned}
& x_{3}(s)=\frac{2}{\sigma} A_{3} \cos \left(\theta_{3}\right)+\frac{1}{\sigma}\left(B_{3}-\mu\right) \sin \left(\theta_{3}\right) \\
& z_{3}(s)=\frac{2}{\sigma} A_{3} \sin \left(\theta_{3}\right)-\frac{1}{\sigma}\left(B_{3}-\mu\right) \cos \left(\theta_{3}\right) .
\end{aligned}
$$

One can get the solution curves corresponding to $\kappa_{4}(s), \theta_{4}(s)$ in a similar fashion by plugging them into (7). Let us give an example in this case as well. Figure 3 represents the solution curve and the phase portrait for the case where $P(\kappa)$ has roots $\{-4,-2,0,6\}$. As in all presented cases we can substitute $s$ with $s-\lambda t$ to get the motion in the plane, which is described in [5]. Therefore we describe all possible motions which come from the mKdV equation with $x=s-\lambda t$. This is an improvement of results [5] because we get analytical solutions for all possible choices for the roots of $P(\kappa)$.

In conclusion we want to summarize the above results. We establish a relation between equilibrium shapes of fluid membranes and the traveling wave solutions 
of the $\mathrm{mKdV}$ equation. Both problems lead to the same ordinary differential equation. We present the solutions in analytical form for all relevant cases. Our considerations unify and extend the work done in [5] and [8]. We also present the corresponding phase portraits.

\section{Further Developments}

Let us try to generalize the ideas in the present paper for non-cylindrical surfaces immersed in $\mathbb{R}^{3}$. For simplicity instead of the Helfrich energy functional $\mathcal{F}_{S}$, we use the Willmore functional for an immersed surface (smooth and orientable) $S \subset$ $\mathbb{R}^{3}$

$$
\mathcal{W}_{S} \equiv \int_{S}\left(H^{2}-K\right) \mathrm{d} A=\frac{1}{4} \int_{S}\left(\kappa_{1}-\kappa_{2}\right)^{2} \mathrm{~d} A
$$

where $\kappa_{1}, \kappa_{2}$ are the principle curvatures of $S$ and $H=1 / 2\left(\kappa_{1}+\kappa_{2}\right), K=\kappa_{1} \kappa_{2}$. Here we think of $S$ as map from some two-dimensional manifold $M$ into $\mathbb{R}^{3}$.

The Euler-Lagrange equation for this energy functional is well known (e.g. see [9]) and is as follows

$$
\Delta_{S} H+2\left(H^{2}-K\right) H=0
$$

where $\Delta_{S}$ is the Laplace-Beltrami operator on the surface $S$.

Setting $\mathcal{M}_{S} \equiv \Delta_{S}+2\left(H^{2}-K\right)=\Delta_{S}+\frac{\left(\kappa_{1}-\kappa_{2}\right)^{2}}{2}$, equation (23) transforms into

$$
\mathcal{M}_{S} H=0 .
$$

Now we give the definition of flow associated to $\mathcal{W}_{S}$. Let $\{S(t) ; t \geq 0\}$ be a family of surfaces immersed in $\mathbb{R}^{3}$. The Willmore flow is defined to be

$$
\frac{\partial S}{\partial t}=\mathcal{M}_{S(t)} H \xi
$$

where $\xi(t)$ is the unit normal vector to $S(t)$. Critical points for (25) are surfaces solving (24). We will mention equation (25) later on.

In this paper we were looking at evolution of planar curves to relate it to a Willmore type functional. To generalize, naturally we want to consider evolution of surfaces immersed in $\mathbb{R}^{3}$. The most studied one is the mean curvature flow. There is an abundance of literature on the subject, for example one can look up [11]. Using the above notation it is given by

$$
\frac{\partial S}{\partial t}=H \xi
$$


If we want to look for surfaces where the mean curvature has critical value we need a formula for the evolution of $H$ under this flow. We have the following result at our disposal (see [11])

$$
\frac{\partial H}{\partial t}=\Delta_{S(t)} H+|A|^{2} H \equiv \mathcal{L}_{S(t)} H .
$$

Here $|A|^{2}=\kappa_{1}^{2}+\kappa_{2}^{2}$ is the norm of the second fundamental form on $S(t)$. The operator $\mathcal{L}_{S}$ is the Jacobi operator coming from the second variation formula for the area of $S(t)$. This Jacobi operator appears in various setups, for example the first author made use of it for problems in capillarity theory (see [4]). Looking for critical points for the mean curvature we get

$$
\mathcal{L}_{S(t)} H=0
$$

Note that for cylindrical surfaces equations (24) and (28) are identical up to a factor of two. The above discussion suggests that we look for a another flow to describe the general case. The condition we will would like to require on the flow is as follows

$$
\frac{\partial H}{\partial t}=\mathcal{M}_{S(t)} H
$$

In our view there are two natural candidates. Obviously, the first one to consider is the Willmore flow (25). We need the compute the evolution of curvature to see if it fits condition (29). Another candidate is hinted by the relation we have with the $\mathrm{mKdV}$ equation. There we had an evolution of planar curves with normal velocity equal to the derivative of curvature with respect to arc length. Therefore we can consider the following flow

$$
\frac{\partial S}{\partial t}=H_{\nu} \xi
$$

Here $H_{\nu}=<\nabla_{S(t)} H, \xi>$, where $\nabla_{S}$ is the surface gradient. Again $\partial H / \partial t$ needs to be computed to see if it resembles (29).

The above discussion also hints it might be worth studying evolutions $\partial S / \partial t=$ $F \xi$, that satisfy $\partial H / \partial t=F$. Possible approach for this would be finding examples and conditions for existence before proceeding with studying other properties for such flows.

\section{References}

[1] Blyth M., Parau E. and Vanden-Broeck J.-M., Hydroelastic Waves on Fluid Sheets, J. Fluid. Mech. 689 (2011) 541-551. 
[2] Helfrich W., Elastic Properties of Lipid Bilayers: Theory and Possible Experiments, Z. Naturforsch 28 (1973) 693-703.

[3] Lawden D., Elliptic Functions and Applications, Springer, New York 1989.

[4] Marinov P., Stability of Capillary Surfaces with Planar Boundary in the Absence of Gravity, Pac. J. Math. 255 (2012) 177-190.

[5] Nakayama K. and Wadati M., Motion of Curves in the Plane, J. Phys. Soc. Jap. 62 (1993) 473-479.

[6] Nishinari K., Nonlinear Dynamics of Solitary Waves in an Extensible Rod, Proc. R. Soc. Lond. A 453 (1997) 817-833.

[7] Vassilev V., Djondjorov P., Hadzhilazova M. and Mladenov I., Traveling Wave Solutions of the Gardner Equation and Motion of Plane Curves Governed by the $m K d V$ Flow, AIP Conf. Proc. 1404 (2011) 86-93.

[8] Vassilev V., Djondjorov P. and Mladenov I., Cylindrical Equilibrium Shapes of Fluid Membranes, J. Phys.A: Math.\&Theor. 41 (2008) 435201.

[9] Vassilev V. and Mladenov I., Geometric Symmetry Groups, Conservation Laws and Group-Invariant Solutions of the Willmore Equation, In: Geometry, Integrability and Quantization V, I. Mladenov and A. Hirshfeld (Eds), SOFTEX, Sofia 2004, pp 246-265.

[10] Zelikin M., Theory and Applications of the Problem of Euler Elastica, Russ. Math. Surv. 67 (2012) 281-296.

[11] Zhu X., Lectures on Mean Curvature Flows, AMS/IP Series in Advanced Mathematics 32, Providence 2002.

Petko I. Marinov

Experian DA, EMEAI

Sofia 1612, BULGARIA

E-mail address: petko.marinov@experian.com

Ivaïlo M. Mladenov

Institute of Biophysics

Bulgarian Academy of Sciences

Sofia 1113, BULGARIA

E-mail address: mladenoveobzor.bio21.bas.bg 